\title{
Cryptocurrency Blockchain Technology in the Digital Revolution Era
}

\author{
Intan Dwi Astuti ${ }^{1}$, Suryazi Rajab ${ }^{2}$, Desky Setiyouji ${ }^{3}$ \\ Management Information System ${ }^{1}$, Technical Information ${ }^{2,3}$ \\ University of Raharja ${ }^{1,2,3}$ \\ e-mail: intan.dwi@raharja.info1', Suryazi.rajab@raharja.info², desky@raharja.info3
}

Astuti, I. D., Rajab, S., \& Setiyouji, D. (2022). Cryptocurrency Blockchain Technology in the Digital Revolution Era. Aptisi Transactions on Technopreneurship (ATT), 4(1).

DOI: https://doi.org/10.34306/att.v4i1.216

Author Notification 26 Oktober 2021 Final Revised 20 December 2021 Published 05 January 2022

\begin{abstract}
Cryptocurrency is a blockchain-based technology that is often used as a digital currency. Digital money is different from conventional money, this type of money does not have a physical form but only a block of data bound by a hash as validation. The data is spread to every cryptocurrency user who is in the environment. So that when a user makes a transaction, data mining will be carried out. Cryptocurrencies have several advantages but also have disadvantages when used as currency, from a legal point of view there is still no law governing the circulation of currency in digital form (cryptocurrency). The government must recognize digital currency so that it can be accepted by the general public as a means of payment. For now, the government has not recognized digital money (cryptocurrency) as a currency, because Cryptocurrency is a new phenomenon by some people in Indonesia. The rapid development of technology in the 4.0 revolution era, in the next few years money in physical form will be replaced by digital money, seeing the many conveniences provided by using digital money.
\end{abstract}

Keywords: Cryptocurrency, Blockchain, Digital Revolution Era 4.0, Digital Money, Digital Money.

\section{Introduction}

The use of technology in the era of the digital revolution has made technology a major need, plus science and technology that are developing so fast make technology in this era used in all fields of science. In the field of money economics, there is one means of payment that is used when making transactions, whether in the form of transactions for goods or services, but with technological advances, a term called digital transactions has emerged, people who want to make transactions can transact without having to bring their money, only it is enough to save their money in a bank and then people can make transactions either using mobile banking, ATMs, or by using more sophisticated technology. Along with the increasing technology in processing transactions, the term E-Commerce began to emerge [1].

E-Commerce is an activity related to buying and selling using mobile devices or computers and the internet network as a medium. One of the digital transactions that is on the rise is bitcoin which is one of the money in the form of digital encryption or often called cryptocurrency. The validation of transaction data used in using digital money is blockchain
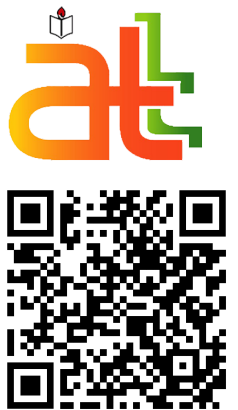
technology [2]. Blockchain is a block of data that is interconnected so that it looks like a chain, in short it will connect users without going through any intermediaries so each user will store each other's data block. The system used on the blockchain can only add data so no data will be changed because each transaction will only add new blocks to each user device in the form of different encrypted data [3].

Based on the 2015 bitcoin journal written by Ferry Mulyanto with the title Utilizing Cryptocurrency as the Application of Rupiah Currency into Digital Form Using Bitcoin Technology (Ferry Mulyanto, 2015), Ferry Mulyanto publishes about the application of Cryptocurrency as a substitute for conventional currency in Indonesia, in his journal saying people need freedom in conducting transactions without being faced with the constraints of different payment systems. Ferry also said that the existence of cryptocurrencies such as bitcoin would solve the problems faced by Bank Indonesia in setting standards for electronic currencies. So people don't have to worry about different currencies when making transactions [4].

Cryptocurrency has several advantages and disadvantages if you want to use it as a currency, for now there is no clear and definite law to regulate the circulation of currency in digital form (cryptocurrency) such as bitcoin for example. If there is an abuse of money in digital form such as fraud, money laundering, or other criminal acts, the institution that will be responsible will not exist [5]. In addition, money that can be used as a means of payment must qualify as a means of payment, and be recognized by the government. Cryptocurrency digital money currently does not meet the requirements, there is no recognition from the government as a means of payment, because Bitcoin is a new phenomenon by some people in Indonesia. However, the rapid and rapid development of technology in the 4.0 revolution era cannot be denied. In the next few years money in physical form will be replaced by digital money [6].

\section{Literature Review}

A. Money Money in general is a medium of exchange that can be used as a legal means of payment in conducting a transaction of goods or services. The function of money is grouped into two types, namely the primary function and the secondary function [7].

a. Primary Function

Money as a primary function is the main function of money which is divided into three, namely:

1. Medium of exchange: Money can be used to buy and sell goods and services. If there is no money, goods must be exchanged through a barter process (goods will be traded for other goods in transactions arranged on the basis of mutual need). For example: If I raise chickens and want to buy cows, I have to find someone who is willing to sell cows for my chickens. Such arrangements are often difficult. But Money removes need from the double coincidence of wants.

2. Unit of account: Money is a common standard for measuring the relative value of goods and services.

3. Stores value: Money is the most liquid asset (Liquidity measures how easily an asset can be spent on buying goods and services). Value for money can be maintained over time. This is an easy way to save wealth.

b. Derivative Functions or Secondary Functions

It refers to the functions of money that complement the main functions. These functions are derived from the main function and, therefore, they are also known as 'Derivative Functions' [8].

The main secondary functions are:

1. Deferred Payment Standard: Money as a deferred payment standard means that money acts as a 'standard' for payments, which have to be made in the future.

2. Storing Value (The Asset Function of Money): Money as a store of value means that money can be used to transfer purchasing power 
from the present to the future. Money is a way to store wealth. Although wealth can be stored in other forms as well, money is the most economical and convenient way. It provides security to the individual to meet contingencies, unpredictable emergencies and to repay debts in the future. Under the barter system, it was difficult to use goods as a store of wealth due to the perishability of goods and high storage costs.

B. Blockchain Blockchain is a technology for recording transactions that are interconnected using unique codes in it that are immutable and immutable. How blockchain works When new transactions or edits to existing transactions enter the blockchain, generally most nodes in a blockchain implementation must run an algorithm to evaluate and verify the history of the proposed individual blockchain blocks. If the majority of nodes reach consensus on valid signatures, a new transaction block is accepted into the ledger and a new block is added to the transaction chain. If the majority disapproves of the addition or modification of a general ledger entry, it is rejected and not added to the chain. This distributed consensus model is what allows a blockchain to run as a distributed ledger without the need for multiple authorities, a unifying center telling what transactions are valid and (perhaps more importantly) which are not [9].

C. Cryptocurrency Cryptocurrency is a digital currency built using blockchain technology. This technology does not require a third party as an intermediary. So that every transaction becomes more transparent. Cryptocurrency using blockchain technology, every existing data will be interconnected where every data is owned by everyone who is in the user environment of the cryptocurrency system [10]. Over the past few years, digital currencies have been rapidly gaining public attention, namely:

1. Fraud-proof: When a cryptocurrency is created, all confirmed transactions are stored in a general ledger. All coin owner identities are encrypted to ensure the validity of the records. Because the currency is decentralized, you own it. Neither the government nor the banks have control over it.

2. Identity Theft: The ledger ensures that all transactions between "digital wallets" can calculate an accurate balance. All transactions are checked to ensure that the coins used are owned by the current spenders. This general ledger is also referred to as a "transaction blockchain". Blockchain technology ensures secure digital transactions through encryption and "smart contracts" which makes the entity completely uncontrollable and fraud free.

3. Instant Settlement: Blockchain is the reason why cryptocurrencies have any value. Ease of use is the reason why cryptocurrencies are in great demand. All you need is a smart device, an internet connection and you'll soon be your own bank making payments and money transfers.

4. Accessibility: There are more than two billion people with access to the Internet who do not have the right to use traditional exchange systems. These guys are cryptic clues to cryptic.

5. You are the owner: There is no other electronic money system where your account is owned by you.

D. Hash

The network of users, spread all over the world and not tied to each other, be it in personal or professional ties, and directly receive the latest transaction data. They run the data through a cryptographic algorithm, which generates a "hash", a string of numbers and letters that serves to verify the validity of the information, but does not reveal information from the data itself [11].

Example hash AG53HD6VD736WG8S7A81KQ, you can't find out the transaction relevant to the block (\#480504). Either way you can take the set of data claiming to be block 
\#480504 and make sure it's not tampered with. If one number is out of place, it doesn't matter if it's not significant, the data will result in a completely different hash. If you run the declaration of freedom using a hash calculator, the result you will get is AG53HD6VD736WG8S7A81KQ. Using this technology, it is possible for the bitcoin network to be able to check the validity of blocks. It takes a long time to go through the entire ledger to make sure that the transaction pool user hasn't tried anything interesting. Instead, the hash of the previous block appears in the new block. If every minute the details are changed in the previous block, the hash will change. In fact, if each change reaches 20,000 blocks back down the chain, that block hash will trigger a new set of hashes and release the network [12].

\section{Discussion}

Cryptocurrency uses a technology similar to a chain as a system for recording each transaction, this technology is often known as blockchain. Blockchain technology allows all its members to be connected to each other without going through a third party. So that all transaction systems become more transparent [13].

a. Terms of using Blockchain (Cryptocurrency) To be able to carry out transactions using blockchain technology [14]. There are several things that must be met, namely:

1. Agreement not to use transactions using banks or third parties.

2. At least 3 people must join or agree to use digital money in the form of cryptocurrency (blockchain)

b. Blockchain (Cryptocurrency) Working Principle Blockchain technology binds every transaction like a chain that is connected to each other and no third party intervenes [15]. As for how blockchain works

1. Transaction data folder that uses cryptocurrency by utilizing blockchain technology. Each user will have a folder as a data store for each transaction. This folder will contain data for every transaction, both transactions carried out by yourself and by others, which are in the form of data records where this collection of data will form a registry that will track transactions [16].

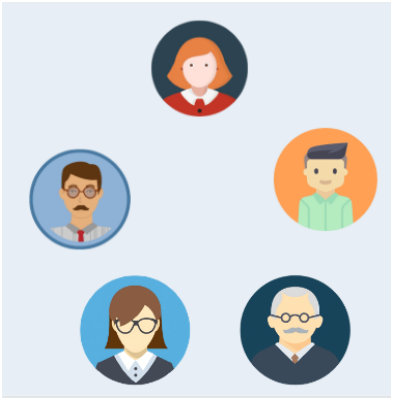

Figure 1. User Transaction

2. Data transaction When there is a transaction to be made. Each user in the chain will record and save it as a new record so that if there is a re-transaction, a new data record will be added to the folder of each user on each device. To perform data transactions, here we assume that we have 5 users who agree to use the cryptocurrency blockchain system [17]. 


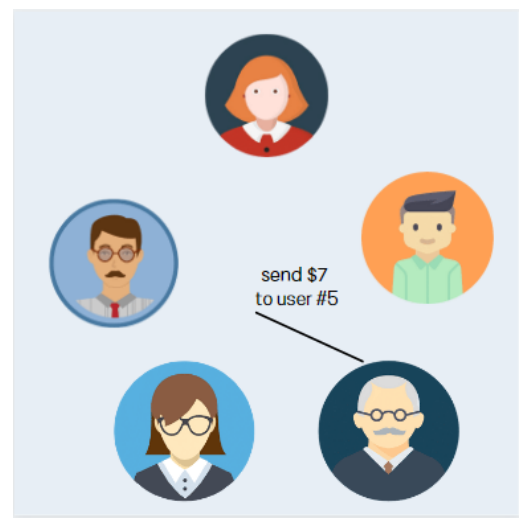

Figure 2. Transaction Flow

User 1 wants to make a transaction against User \#9. User \#1 sends $\$ 5$ to user \#9 To make a transaction user one notifies all members. I want to transfer $\$ 5$ to user \#9. So all will record it in their respective folders. All members check whether user \#1 has sufficient balance to make a transaction of $\$ 5$. If it is valid then the transaction is successful, all members record back in their respective folders. The transaction was successful.

3. Data Validation To validate data on the cryptocurrency blockchain, a hash function is applied. The hash data function will be created when the transaction is considered valid. As in the picture of the transaction between user 2 and user 9 . After checking the balance data held by user 2 on another user's record and the balance is declared valid then the transaction can be carried out, the $\$ 5$ dollar balance belonging to user 2 will move to user 9 as a transaction lock then also a data hash generated based on the transaction. For example, the hash is formed from id, seconds, minutes, date, money balance and added a unique code [18]. The hash to be formed for example "AG53HD6VD736WG8S7A81KQ"

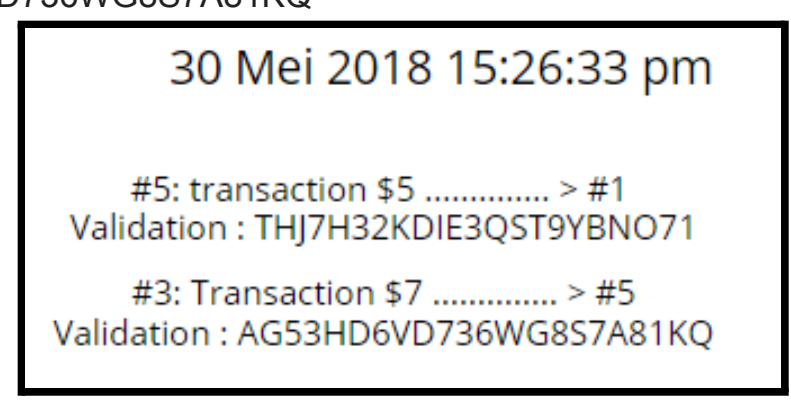

Gambar 3. Transaction Note

c. The Risks of Using Cryptocurrency

Although the use of cryptocurrency has many benefits and advantages, it does not mean that the use of cryptocurrency technology does not have risks. For now, the use of cryptocurrencies such as bitcoin, for example, does not yet have regulations or laws that clearly regulate cryptocurrencies and changes in the value of cryptocurrencies such as bitcoin which tend to be unstable, making it difficult to determine the value. The impact that will arise in the future if this currency does not have standard rules and definitely it is not impossible that fraudulent investments will appear that use cryptocurrency as an exchange rate. However, all of this can still be overcome with legal and standard regulations and strict actions to regulate the use of cryptocurrency digital money so that there is no abuse of the possible adverse effects 
that may occur. As well as seeing the many benefits and conveniences provided in transacting using cryptocurrency digital currencies [19].

d. Analysis of the Use of Cryptocurrency Technology

Based on the results of the presentation in the previous sub-chapter, the researcher can convey any findings obtained from the test results. The transaction process using Cryptocurrency to implement it as a digital currency still needs many conditions that must be met. Especially agreement from all parties is required before using cryptocurrency as a transaction tool. The use of cryptocurrency when it is safe to use because the data on the user cannot be changed, when the data is changed, a hash validation check will be made to all users. Each transaction will generate a new hash which is then recorded on each user. Cryptocurrencies cannot be counterfeited and transactions cannot be arbitrarily reversed by the sender like on a credit card. Cryptocurrencies have many benefits and advantages, but currently there are no standard rules governing their use, there are no laws or regulations that regulate for sure, so this will allow the impact of fraudulent investments that use cryptocurrencies as an exchange rate [20].

\section{Conclusion}

Cryptocurrency is a digital currency using blockchain technology. To be able to use Cryptocurrency, there must be an agreement to use Cryptocurrency without using other third parties. There is a minimum number of 3 members who agree to use the cryptocurrency system. Transaction processes using Cryptocurrencies are stored and recorded in the folder of each member using it. Notes can only be added and cannot be changed or deleted arbitrarily. Every transaction made will generate a Hash which is used as transaction data validation. Transactions using Cryptocurrencies currently there is no law that is absolutely certain to regulate it. Henceforth, it is necessary to do an approach or research on the law of digital money. If there is a law that can lead to regulating digital money in the form of Cryptocurrencies, of course this will have a lot of positive impacts for the community, especially on transactions made through digital media.

\section{References}

[1] T. Wahyuningsih, F. P. Oganda, and M. Anggraeni, "Design and Implementation of Digital Education Resources Blockchain-Based Authentication System," Blockchain Front. Technol., vol. 1, no. 01, pp. 74-86, 2021.

[2] U. Rahardja, Q. Aini, and A. Khoirunisa, "Effect of iDu (iLearning Education) on Lecturer Performance in the Lecture Process," Aptisi Trans. Manag., vol. 2, no. 2, pp. 140-148, 2018.

[3] H. Haris and N. Priliasari, "THE DESIGN OF WEB-BASED TRAINING MANAGEMENT INFORMATION SYSTEMS AT PT. SINTECH BERKAH ABADI," ADI J. Recent Innov., vol. 2, no. 2, pp. 269-274, 2020.

[4] U. Rahardja, N. Lutfiani, and S. Amelia, "Creative Content Marketing In Scientific Publication Management In Industrial Era 4.0," Aptisi Trans. Manag., vol. 3, no. 2, pp. 168-178, 2019.

[5] R. Geethanjali, "Notice of Retraction Survey on Health Monitoring of Elderly Using loT," Aptikom J. Comput. Sci. Inf. Technol., vol. 2, no. 3, pp. 131-136, 2017.

[6] N. Azizah, D. Supriyanti, S. F. A. Mustapha, and H. Yang, "The Role of Web Based Accounting Online System 2.0 as the Company's Income and Expense Management," Aptisi Trans. Manag., vol. 1, no. 1, pp. 44-49, 2017.

[7] M. Yusup, R. S. Naufal, and M. Hardini, "Management of Utilizing Data Analysis and Hypothesis Testing in Improving the Quality of Research Reports," Aptisi Trans. Manag., vol. 2, no. 2, pp. 159-167, 2018.

[8] S. Watini, Q. Aini, M. Hardini, and U. Rahardja, "Improving Citizen's Awareness in Conserving Diversity of Malay Traditional Dances in Malaysia through the Art 
Appreciation Performed by Students of Early Childhood Education Study Program," Int. J. Psychosoc. Rehabil., vol. 24, no. 8, pp. 2730-2737, 2020, doi: 10.37200/IJPR/V24I8/PR280292.

[9] P. Pirnay-Dummer, "Knowledge and Structure to Teach: A Model-Based Computer-Linguistic Approach to Track, Visualize, Compare and Cluster Knowledge and Knowledge Integration in Pre-Service Teachers," in International Perspectives on Knowledge Integration, Brill Sense, 2020, pp. 133-154.

[10] A. Philip, C. S. Putri, and P. M. Arifanggi, "Traffic Light Timer Control Using Raspberry Pi," Aptisi Trans. Technopreneursh., vol. 1, no. 2, pp. 134-143, 2019.

[11] P. Padeli, M. Mulyati, M. Faisal, and S. Debora, "E-CRM Mobile Applications To Improve Customer Loyalty (Case Study: PT Supermal Karawaci)," Aptisi Trans. Manag., vol. 4, no. 1, pp. 41-48, 2020.

[12] N. L. P. G. S. Kusuma, P. E. T. Dewi, and N. P. R. K. Sari, "Regulation of Copyright Certificate as a Material Guarantee and Bankrupt Estate/Beodel in Indonesia," ADI J. Recent Innov., vol. 2, no. 2, pp. 290-303, 2020.

[13] D. Ahmad, H. D. Ariessanti, and K. Awaliyah, "Implementation of E-Commerce Website to Increase Online Sales of Case Study of Baby Wise BSD Tangerang," Aptisi Trans. Manag., vol. 1, no. 1, pp. 11-16, 2017.

[14] P. Ramanathan, "Implementation of PC Controlled Wireless Video Transmitting Vehicle," Aptikom J. Comput. Sci. Inf. Technol., vol. 2, no. 2, pp. 63-67, 2017.

[15] J. Leonard, D. Damanik, and O. Amirkhasanah, "Application of Information Session Information System as Media Submission of Final Results Comprehensive Session," J. Recent Innov., vol. 1, no. 1, pp. 62-70, 2020.

[16] I. B. H. Murtiarso, Y. I. Graha, and N. Alnabawi, "Analysis of the Role of Quality on Job Satisfaction in Management with Sampling Techniques," Aptisi Trans. Manag., vol. 2, no. 1, pp. 28-36, 2018.

[17] B. S. Riza, M. Y. Mashor, and E. V. Haryanto, "THE APPLICATION OF RSA AND LSB IN SECURITY OF MESSAGES ON IMAGERY," ADI J. Recent Innov., vol. 1, no. 1, pp. 20-32, 2019.

[18] H. Hady, H. Henderi, and D. M. Putri, "The Application of Science Concepts in Management Research Reports Based on Scientific Truth," Aptisi Trans. Manag., vol. 2, no. 1, pp. 37-44, 2018.

[19] E. S. Aisyah, E. P. Harahap, and N. Salsabila, "The Effect Requirements Selling In The Marketplace For Security Against Buyer Trust," Aptisi Trans. Manag., vol. 4, no. 1, pp. 67-75, 2019.

[20] U. Rahardja, T. Hariguna, and W. M. Baihaqi, "Opinion mining on e-commerce data using sentiment analysis and k-medoid clustering," Proc. - 2019 12th Int. Conf. Ubi-Media Comput. Ubi-Media 2019, pp. 168-170, 2019, doi: 10.1109/Ubi-Media.2019.00040. 